\title{
Estudo das propriedades físico-químicas da glibenclamida e excipientes que influem sobre resultados do ensaio de dissolução para medicamento similar e genérico
}

\author{
Effect of the physicochemical properties of glyburide on the results of \\ dissolution test for generic and similar drugs
}

RIALA6/1580

Euclides Quintino da Silva FILHO ${ }^{1 \star}$, José Luiz Neves de AGUIAR ${ }^{1}$, André Luis Mazzei

ALBERT $^{1}$, Armi Wanderley NOBREGA ${ }^{2}$

*Endereço para correspondência: ${ }^{1}$ Laboratório de Medicamentos, Cosméticos e Saneantes, Departamento de Química, Instituto Nacional de Controle de Qualidade em Saúde, Fundação Oswaldo Cruz.Av. Brasil, 4365, Manguinhos, Rio de Janeiro, RJ, Brasil, CEP 21.040-900. Tel:(21)3865-5228. Email: euclides.filho@incqs.fiocruz.br

${ }^{2}$ Assessoria técnico-científico, Diretoria, Instituto Nacional de Controle de Qualidade em Saúde, Fundação Oswaldo Cruz Recebido: 16.08.2013 - Aceito para publicação: 29.11.2013

\begin{abstract}
RESUMO
Alguns insumos farmacêuticos ativos (IFA) possuem como característica, a possibilidade de apresentarem o polimorfismo, que pode se desenvolver em alguma das etapas do processo de produção na indústria. No caso deste não ser caracterizado e especificado, um diferente polimorfo poderá ser utilizado equivocadamente durante o processo de fabricação. A ocorrência de polimorfismo pode originar importantes variações nas propriedades físico-químicas dos IFAs, principalmente quanto à solubilidade. Alguns medicamentos de glibenclamida (GLIB) apresentaram denúncias de ineficácia terapêutica e a presença de polimorfos pode ser uma das possíveis causas. Neste trabalho foram analisados cinco medicamentos e cinco IFAS de diferentes fornecedores. Para os medicamentos foram feitos testes característicos de verificação de equivalência farmacêutica. Nos IFAS, as diversas técnicas empregadas não evidenciaram presença de polimorfos ou alterações importantes nas propriedades físico-químicas e na velocidade de dissolução intrínseca. Entretanto, os perfis de dissolução dos medicamentos, principalmente, entre os dois similares A e $\mathrm{B}$ demonstraram diferenças apontadas pelos valores do fator $f_{2}$, respectivamente, de 20 e 42 , os quais indicaram associação destes valores com a presença de distintos excipientes, como por exemplo o manitol e diferentes processos de produção industrial.
\end{abstract}

Palavras-chave. glibenclamida, dissolução, similar, polimorfismo.

\begin{abstract}
Some active pharmaceutical ingredients (API) might present polymorphism at any stage of the industry production process. In caseit is not characterized and specified, a different polymorph might be erroneously used during the manufacturing procedure. Polymorphisms cause some variations in the physicochemical properties of APIs, especially in solubility. Therapeutic inefficacy was detected in some glyburide drug products, and the occurrence of polymorphs might be one of the possible reasons. This study analyzed five drug products and five APIs., The characteristic pharmaceutical equivalence tests were used for analyzing the drug products. The techniques employed to evaluate the APIs showed no differences in polymorphism, no significant changes in the physicochemical properties or in the intrinsic dissolution rate. However, the dissolution profiles of the drug products, mainly between two similar products $\mathrm{A}$ and $\mathrm{B}$, showed significant differences in the $f_{2}$ factor values, being 20 and 42, respectively, indicating that these values were related to the occurrence of different excipients, such as mannitol.
\end{abstract}

Keywords. glyburide, dissolution, similar, polymorphism. 
Filho EQS, Aguiar JLN, Albert ALM, Nobrega AW. Estudo das propriedades físico-químicas da glibenclamida e excipientes que influem sobre resultados do ensaio de dissolução para medicamento similar e genérico. Rev Inst Adolfo Lutz. São Paulo, 2013; 72(4):316-21.

\section{INTRODUÇÃO}

A glibenclamida (GLIB), representação estrutural Figura 1, pertencente à segunda geração do grupo das sulfoniluréias, atua estimulando a liberação de insulina através do bloqueio dos canais do potássio nas células ß pancreáticas sendo indicada para o controle da glicemia em pacientes portadores da diabete tipo 2 (dois) ou diabetes mellitus não-insulino-dependente ${ }^{1}$. Entre as propriedades físico-químicas da GLIB estão: baixa solubilidade em água e éter etílico, solubilidade em dimetilformamida, pouco solubilidade em etanol, metanol e clorofórmio, dissolvendo-se em soluções diluídas de hidróxidos alcalinos ${ }^{2}$. Possui coeficiente de partição octanol / água $(\log \mathrm{P}) 4,8$, pKa $5,3^{3}$ e ponto de fusão na faixa de: $169-174{ }^{\circ} \mathrm{C}^{4}$.

Dependendo do solvente utilizado, a cristalização da GLIB pode dar origem a formas polimórficas e pseudo-polimórficas, as quais são significativamente diferentes com respeito à solubilidade e propriedades de fusão ${ }^{5,6}$. Um polimorfo, obtido a partir da tentativa de elucidação da transição vítrea pelo aquecimento, resfriamento e reaquecimento, resultou em baixo valores de dissolução e biodisponibilidade em comprimidos ${ }^{7}$. O estudo das propriedades termodinâmicas, de sistemas obtidos a partir da cristalização das formas I e IV, que são as descritas na literatura, quando utilizados diferentes solventes sugerem a existência de outras formas polimórficas ${ }^{8}$.

Teoricamente, os diversos polimorfos possuem diferentes velocidades de dissolução. Em alguns casos a diferença não é significativa, entretanto quando ocorre o oposto, os medicamentos podem tornar-se menos ativos, inativos ou tóxicos, quando existe uma relação direta entre esse parâmetro e a atividade farmacológica ${ }^{9}$. Alguns estudos demonstram que absorção oral da GLIB é dependente da sua formulação ${ }^{10}$, e que o comportamento

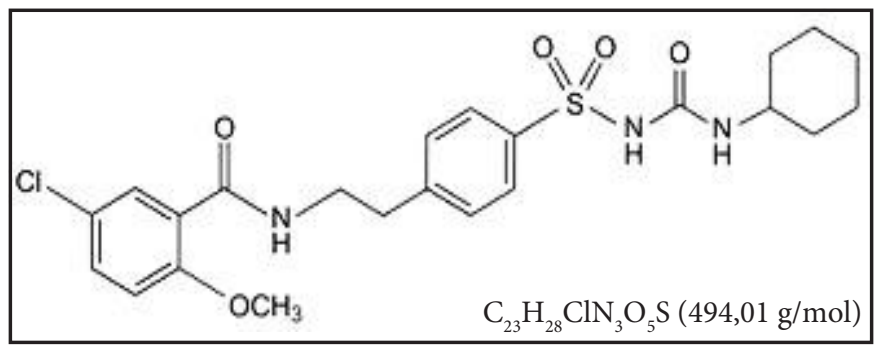

Figura 1. Representação estrutural da Glibenclamida (5-Cloro-N-[2[4-[[[(cicloexilamino) carbonil]amino]sulfonil]fenil]etil]-2-metoxibenzamida) de diferentes formulações podem apresentar importantes diferenças no desempenho oral e consequentemente na biodisponibilidade de medicamento ${ }^{11}$.

Os dados do Sistema de Gerenciamento de Amostras do Instituto Nacional de Controle de Qualidade em Saúde (INCQS), no período de 2000 a 2008, apontaram que 329 registros das solicitações de análises fiscais continham denúncia de suspeita de ineficácia terapêutica, inclusive cinco medicamentos de GLIB, podendo o polimorfismo ser uma de suas possíveis causas. Portanto, identificou-se a necesidade de aprofundar os estudos sobre a determinação de polimorfos em fármacos.

Neste contexto o objetivo deste trabalho foi a determinação de polimorfos em fármacos, tendo como objeto de estudo a GLIB. Especificamente correlacionar a velocidade de dissolução intrínseca dos fármacos, com os perfis de dissolução dos produtos e paralelamente comparar o fator de similaridade $\left(f_{2}\right)$ dos produtos, assim como avaliar o poder discriminante do teste de dissolução in vitro para as diferentes formulações dos produtos de GLIB.

\section{MATERIAL E MÉTODOS}

\section{Amostragem}

Foram analisados cinco amostras de comprimidos contendo $5 \mathrm{mg}$ de GLIB e os insumos farmaceuticos ativos puros provenientes de dois fabricantes de generico, dois de similar e o referência. O padrão foi GLIB, Substância Química de Referência da Farmacopeia Brasileira, teor $99,96 \%$, lote 1018. As análises foram realizadas no Laboratório de Medicamentos, Cosméticos e Saneantes do Departamento de Química do INCQS/Fiocruz e no Laboratório de Estudo de Estado Sólido do Instituto de Tecnologia em Fármacos de Farmanguinhos/Fiocruz.

\section{Técnicas empregadas}

I) Distribuição de tamanho de partículas (Mastersizer Malvern): meio de dispersão composto por água e o agente dispersante Tween $80^{\circ}$ (SigmaAldrich); tempo de leitura das amostras de 10 segundos e intensidade do laser de 82,7 \%. II) Microscopia Óptica (Olympus BX50): foi utilizado um aumento de 500 vezes. III) Varredura Exploratória Diferencial (Mettler Toledo 822): sob atmosfera dinâmica de nitrogênio com vazão de $80 \mathrm{~mL} / \mathrm{min}$ e razões de aquecimento de 40 e $5 \mathrm{~K} / \mathrm{min}$, no intervalo de temperatura de 25 a 300 K. IV) Difratometria 
Filho EQS, Aguiar JLN, Albert ALM, Nobrega AW. Estudo das propriedades físico-químicas da glibenclamida e excipientes que influem sobre resultados do ensaio de dissolução para medicamento similar e genérico. Rev Inst Adolfo Lutz. São Paulo, 2013; 72(4):316-21.

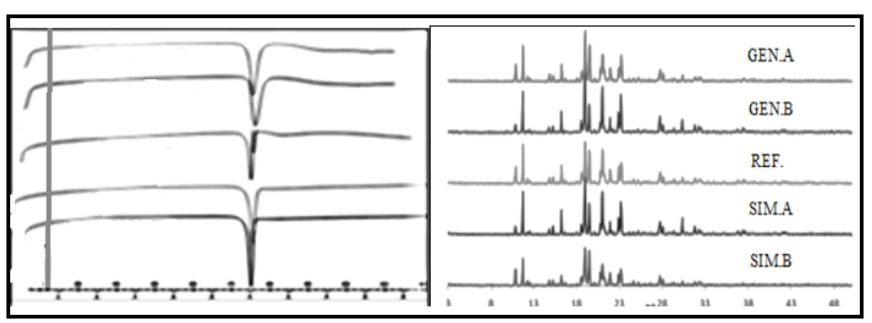

Figura 2. Curvas de Varredura Exploratória Diferencial e de Difratogramas de Raios X de pós dos fármacos de GLIB

de Raios-X (Bruker D8-Advanced): operação a 40 kV e 40 $\mathrm{mA}$, com velocidade de varredura de $0,05 \%$ segundo no intervalo $2^{\theta}$ de 3 a $50^{\circ}$. V) Ponto de fusão (Büchi B-540): em capilar de vidro para determinação da faixa de fusão pelo método capilar. VI) Termogravimetria (Mettler Toledo 851): sob atmosfera dinâmica de nitrogênio com vazão de $50 \mathrm{~mL} / \mathrm{min}$ e razão de aquecimento de $10 \mathrm{~K} /$ min, no intervalo de temperatura de 25 a $300 \mathrm{~K}$. VII) Ensaio de velocidade de dissolução intrínseca dos IFAS (Hanson SR-8), o coeficiente VDI foi determinado de acordo com uma aparelhagem similar à descrita na USP. Para o preparo das pastilhas, aproximadamente $500 \mathrm{mg}$ de cada fármaco foi pesado (Mark 3100) diretamente na matriz do pastilhador (Pastilhador de aço inoxidável Perkin-Elmer). Após a colocação de punção, aplicouse a força de compressão de uma tonelada pelo tempo de um minuto (Prensa Hidráulica Carver 3912). Esse conjunto, contendo o sólido compactado, foi então, preso à haste do aparato $\mathrm{n}^{\circ} 1$ (cesta) do dissolutor. A haste é posicionada a $2,5 \mathrm{~cm}$ do fundo da cuba, observando à

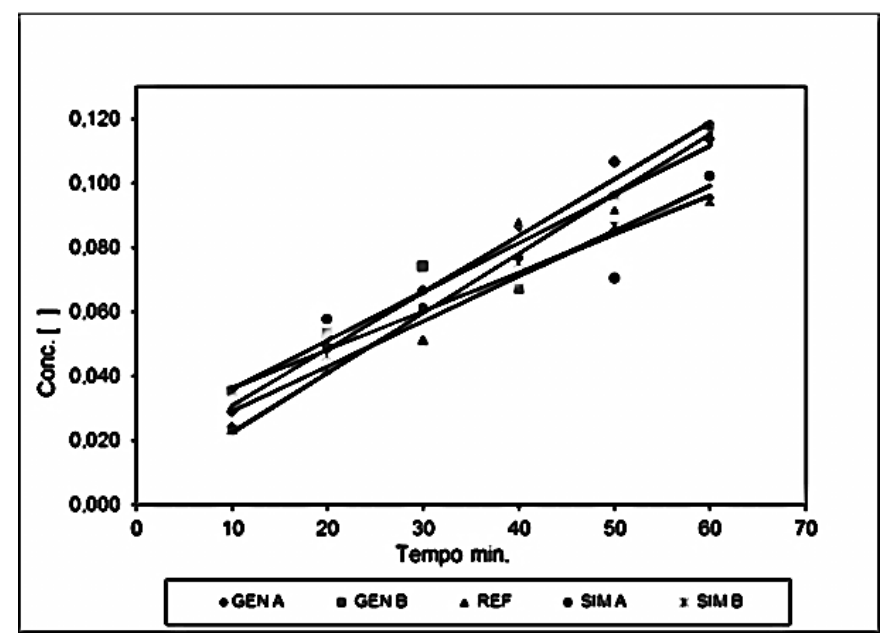

Figura 3. Velocidade de dissolução intrínseca dos fármacos Equação das retas: GEN A, $y=0,0009 x+0,00115$; GEN B, $y=0,0008 x+0,0073 ; \quad$ SIM A, $y=0,0008 x+0,0063 ; \quad$ SIM $B$, $y=0,0008 x+0,0076$; REF, $y=0,0008 x+0,0044$ não formação de bolhas sobre a superfície do material em contato com o líquido. A coleta das amostras foi feita de 20 em 20 minutos durante 120 minutos. Em condições de área superficial constante, os valores de concentração dissolvida devem situar-se sobre uma reta. A inclinação da reta produzida exprime o valor do coeficiente de dissolução intrínseca ${ }^{12}$. VIII) Ensaio de dissolução de comprimidos de GLIB (Hanson SR-8) as condições seguidas foram as do ensaio de dissolução e de doseamento de GLIB descrito na FB 20104. Os comprimidos foram adicionados individualmente às cubas de dissolução (com intervalos de um minuto entre as adições) que continham $900 \mathrm{~mL}$ de tampão fosfato $\mathrm{pH} 7,30 \pm 0,02$ como meio de dissolução na temperatura de $37,0 \pm 0,5^{\circ} \mathrm{C}$, convenientemente degaseificado. No momento da adição dos comprimidos (tempo zero), inicia-se a agitação do meio mediante pás rotatórias, aparato $\mathrm{n}^{\circ} 2$, com velocidade pré-fixada de $75 \mathrm{rpm}$ até o tempo limite do ensaio, que no caso, corresponde a 60 minutos. IX) As análises de cromatografia líquida de alta eficiência (CLAE) foram realizadas em um cromatógrafo líquido Dionex composto de detector ultravioleta a 230 nanômetros e injetor automático. $\mathrm{O}$ volume injetado foi de $100 \mu \mathrm{L}$. A coluna analítica usada foi Nova-Pak ${ }^{\circledast} \mathrm{C} 8$, $4 \mu \mathrm{m},(150 \times 4,6) \mathrm{mm}$. A fase móvel foi uma mistura de tampão fosfato monobásico de potássio (Vetec) $\mathrm{pH}$ 3,0 (Micronal B474) e acetonitrila (Merck) (47:53), preparada como descrito na FB 2010. Fluxo da fase móvel foi de $1,5 \mathrm{~mL} / \mathrm{min}$.

\section{RESULTADOS E DISCUSSÃO}

A pesquisa das propriedades físico-químicas através dos testes executados neste trabalho, apontaram ausência de polimorfismo nos fármacos de GLIB. Observando a Figura 2, ao lado esquerdo está o termograma proveniente do ensaio de varredura exploratória diferencial, onde os perfis energéticos das amostras de GLIB foram similares entre si nas condições analíticas utilizada e à direita o difratograma proveniente do ensaio de difratometria de raios-X em pó mostra que as mesmas correspondem à forma cristalina I descrita em Rodríguez et $\mathrm{al}^{8}$. Para determinação e identificação de polimorfismo em fármacos, estas técnicas podem estar entre as principais, pois podem confirmar possíveis resultados encontrados.

A velocidade de dissolução intrínseca (VDI), definida como velocidade com que substâncias puras 
Filho EQS, Aguiar JLN, Albert ALM, Nobrega AW. Estudo das propriedades físico-químicas da glibenclamida e excipientes que influem sobre resultados do ensaio de dissolução para medicamento similar e genérico. Rev Inst Adolfo Lutz. São Paulo, 2013; 72(4):316-21.

Tabela 1. Avaliação estatística (teste ANOVA) das curvas de VDI

\begin{tabular}{|c|c|c|c|c|c|c|}
\hline \multirow{3}{*}{ tempo } & \multicolumn{6}{|c|}{ Curva de Velocidade de dissolução intrínseca } \\
\hline & \multirow{2}{*}{$\begin{array}{c}\mathrm{mg} / \mathrm{cm}^{2} \cdot \mathrm{min}^{-1} \\
\text { Gen } 1\end{array}$} & \multirow{2}{*}{$\begin{array}{c}\mathrm{mg} / \mathrm{cm}^{2} \cdot \mathrm{min}^{-1} \\
\text { Gen2 }\end{array}$} & \multirow{2}{*}{$\begin{array}{c}\mathrm{mg} / \mathrm{cm}^{2} \cdot \mathrm{min}^{-1} \\
\operatorname{Sim} 1\end{array}$} & \multirow{2}{*}{\multicolumn{2}{|c|}{$\begin{array}{c}\mathrm{mg} / \mathrm{cm}^{2} \cdot \mathrm{min}^{-1} \\
\operatorname{Sim} 2\end{array}$}} & \multirow{2}{*}{$\begin{array}{c}\mathrm{mg} / \mathrm{cm}^{2} \cdot \mathrm{min}^{-1} \\
\text { Ref }\end{array}$} \\
\hline & & & & & & \\
\hline 20 & 0,0271 & 0,02023 & 0,02023 & \multicolumn{2}{|c|}{0,02301} & 0,0206 \\
\hline 40 & 0,04359 & 0,04088 & 0,04157 & \multicolumn{2}{|c|}{0,03992} & 0,03569 \\
\hline 60 & 0,06562 & 0,05548 & 0,05413 & \multicolumn{2}{|c|}{0,0562} & 0,0546 \\
\hline 80 & 0,08481 & 0,07197 & 0,08835 & \multicolumn{2}{|c|}{0,07306} & 0,0732 \\
\hline \multicolumn{7}{|c|}{$\begin{array}{c}\text { Anova: fator único } \\
\text { RESUMO }\end{array}$} \\
\hline Grupo & \multicolumn{2}{|l|}{ Contagem } & & Média & \multicolumn{2}{|c|}{ Variância } \\
\hline $\mathrm{mg} / \mathrm{cm}^{2} \cdot \mathrm{min}^{-1} \mathrm{Gen} 1$ & \multicolumn{2}{|l|}{6} & & 0,0071128333 & \multicolumn{2}{|c|}{0,001028722} \\
\hline $\mathrm{mg} / \mathrm{cm}^{2} \cdot \mathrm{min}^{-1} \mathrm{Gen} 2$ & \multicolumn{2}{|l|}{6} & & 0,062751667 & \multicolumn{2}{|c|}{0,000881776} \\
\hline $\mathrm{mg} / \mathrm{cm}^{2} \cdot \mathrm{min}^{-1} \operatorname{Ref}$ & 6 & & & 0,062088333 & & 52852 \\
\hline & & & OVA & & & \\
\hline Fonte de Variação & $S Q$ & $g l$ & MQ & $F$ & valor-P & F crítico \\
\hline Entre grupos & 0,000334091 & 4 & 8,35226E-05 & 0,089196501 & 0,985000871 & 2,75871 \\
\hline Dentro dos grupos & 0,023409727 & 25 & 0,000936389 & & & \\
\hline
\end{tabular}

se dissolvem numa área superficial constante, é uma técnica que permite caracterizar a solubilidade de uma substância ativa ou excipiente em um determinado meio.

As retas da VDI dos fármacos estão representadas na Figura 3, os interceptos correspondem à fração de substância dissolvida instantaneamente em contato com o meio de dissolução e às inclinações ou VDI aos gradientes de concentrações dissolvidas por tempo. Todos os valores de VDI foram homogêneos $(8,20 \mathrm{E}-04 \pm 5) \%$.

Os valores utilizados para confecção das curvas de VDI foram comparados estatisticamente pelo teste de ANOVA como demonstrado na Tabela 1. Conclui-se que sendo p-valor $(0,9850)$ maior que o nível de significância, não se consegue provar que ao menos uma das médias é diferente das outras.

Uma vez que variações na dissolução podem alterar a eficácia terapêutica de um medicamento, é mais apropriado estudar a velocidade de dissolução intrínseca (VDI) do que estudar a solubilidade do fármaco (uma propriedade de equilíbrio termodinâmico) durante a avaliação dainfluência do estado sólido no desenvolvimento farmacotécnico e no controle de qualidade ${ }^{9}$. Logo, foi feita a comparação dos resultados do ensaio de VDI dos IFAs com os de dissolução dos medicamentos.

A comparação de perfis de dissolução é útil nos casos em que se deseja conhecer o comportamento de dois medicamentos antes de submetê-los a estudo de biodisponibilidade relativa/bioequivalência. Desenvolver uma nova formulação requer amplo conhecimento sobre as propriedades físico-químicas do fármaco e dos excipientes empregados no processo de fabricação ${ }^{13}$.

Atualmente, para a Anvisa, os perfis de dissolução comparativos são avaliados apenas utilizando-se o cálculo do fator de similaridade $\left(f_{2}\right)$, que corresponde a uma medida de semelhança entre as porcentagens dissolvidas de ambos os perfis, o valor de $\left(f_{2}\right)$ deve estar compreendido entre 50 a $100^{14}$. Os medicamentos similares A e B apresentaram respectivamente os valores de $f_{2}$ iguais a 20 e 42 , apontando o quanto os produtos similares estudados têm comportamento próprio quando comparados ao produto referência como demonstrado na Figura 4 e que as alterações em suas formulações podem, em alguns casos, alterar seu comportamento in vitro, acarretando falha no teste de equivalência farmacêutica. 
Filho EQS, Aguiar JLN, Albert ALM, Nobrega AW. Estudo das propriedades físico-químicas da glibenclamida e excipientes que influem sobre resultados do ensaio de dissolução para medicamento similar e genérico. Rev Inst Adolfo Lutz. São Paulo, 2013; 72(4):316-21.

Tabela 2. Principais excipientes dos produtos

\begin{tabular}{cccccc}
\hline Excipiente & GEN B & GEN A & REF & SIM B & SIM A \\
\hline Manitol (Diluente) & & & & & $\mathrm{x}$ \\
$\begin{array}{c}\text { Amido de milho } \\
\quad \begin{array}{c}\text { Amido } \\
\text { pré-gelatinizado }\end{array}\end{array}$ & $\mathrm{x}$ & $\mathrm{x}$ & $\mathrm{x}$ & $\mathrm{x}$ & \\
Lactose (Diluente) & & & & & \\
Lubrificantes & $\mathrm{x}$ & $\mathrm{x}$ & $\mathrm{x}$ & $\mathrm{x}$ & $\mathrm{x}$ \\
\hline
\end{tabular}

Quando as VDI da IFAS são testadas em condições que podem proporcionar uma alta solubilidade, como no caso estudado e suas curvas de regressão analiticas não são estatisticamente diferentes, pode ser possível correlacionar com os resultados do ensaio de dissolução de medicamentos, desde que os produtos sejam testados em condições semelhantes as utilizads para VDI dos IFAS.

No campo da dissolução, a comparação dos perfis de dissolução possui extensa aplicabilidade durante o processo de desenvolvimento do produto, podendo ser usada para estabelecer a similaridade de formas farmacêuticas de dosagem ${ }^{15}$.

No que concerne a composição dos produtos Tabela 2, o SIM. A apresentou menor percentual de dissolução, tendo como característica a presença de manitol em substituição à excipientes que poderiam gerar glicose. Alguns autores citam o caso de armazenamento de formulações contendo manitol que é um excipiente que reconhecidamente apresenta polimorfismo no qual favoreceu o endurecimento dos comprimidos, ocasionando uma redução da porcentagem de liberação durante a dissolução ${ }^{16}$. Somente após 2003 a ANVISA através da $\mathrm{RDC} \mathrm{n}^{\circ} 134^{17}$, estabeleceu as bases legais para a adequação dos medicamentos similares já registrados; logo, o resultado encontrado abaixo do especificado para o produto SIM B pode estar relacionado ao seu processo de produção.

\section{CONCLUSÃO}

O estudo de polimorfismo em fármacos é um processo que envolve várias técnicas analíticas, as mesmas se complementam sendo muito frequente a necessidade de duas ou mais como as empregadas neste trabalho, para uma determinação com boa margem de segurança, contudo não foram determinados polimorfos nos fámacos de GLIB utlizados neste estudo.

Os valores determinados das velocidades de dissolução intrínseca dos fármacos de GLIB estudados

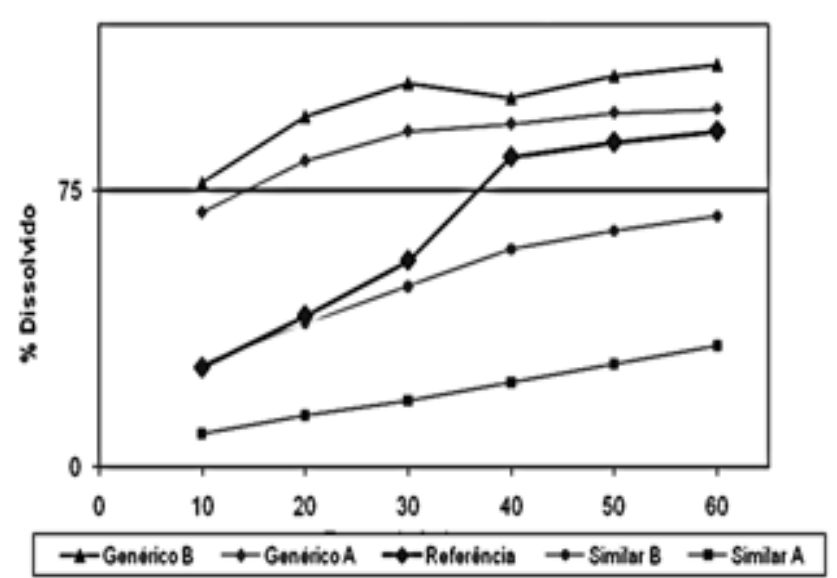

Figura 4. Perfil de dissolução dos medicamentos: Percentagem dissolvida de GLIB em 60 minutos: GEN B= $109 \%$, GEN A= $97 \%$, $\mathrm{REF}=91 \%$, SIM B=68\%, SIM A= $33 \%$

foram praticamente iguais e correlacionando com os resultados distintos dos perfis de dissolução dos produtos é possível determinar que a dissolução in vitro destes produtos é dependente das formulações, dos diferentes processos de produção industrial. e provavelmente das condições do teste de dissolução empregado, principalmente, no caso dos medicamentos similares.

A determinação de polimorfismo em medicamentos, na forma de produto final industrializado, torna-se de dificil interpretação devido a presença de excipientes. Exceção para algumas monografias presentes atualmente em literatura.

O ensaio de dissolução para comprimidos de GLIB da FB apresentou alto poder discriminatório, quando utilizado para comparação dos produtos estudados, com o produto referencia, no teste do fator de similaridade $\left(f_{2}\right)$, para determinação da equivalência farmacêutica dos produtos.

\section{AGRADECIMENTOS}

À Fiocruz / Farmanguinhos / Laboratório de estudo de estado sólido (LESS) pelo apoio na realização deste trabalho.

À Fiocruz / INCQS pela oportunidade e incentivo.

\section{REFERÊNCIAS}

1. Rang HP, Dale MM, Ritter JM. Farmacologia. 4.ed. Rio de Janeiro: Guanabara Koogan; 2001. p.323-329.

2. Merck index: an encyclopedia of chemicals, drugs and biologicals. 14.ed. Whitehouse Station: Merck Research Laboratories, 2006. p.773. 
Filho EQS, Aguiar JLN, Albert ALM, Nobrega AW. Estudo das propriedades físico-químicas da glibenclamida e excipientes que influem sobre resultados do ensaio de dissolução para medicamento similar e genérico. Rev Inst Adolfo Lutz. São Paulo, 2013; 72(4):316-21.

3. Moffat AC, Osselton MD, Widdop B. Clarke's analysis of drugs and poisons. 3.ed. London: Pharmaceutical Press. 2004, v.2. p.1078.

4. Farmacopéia Brasileira. 5.ed. Brasília: ANVISA, 2010. v.2, p.442-5.

5. Suleiman MS, Najib NM. Isolation and Physicochemical characterization of solids forms of glibenclamide. Int $\mathrm{J}$ Pharm. 1989;50:103-9.

6. Hassan MA, AL-hindawi MK, Sallam E, Sheikh Salem M. Preparation and characterization of a new polymorphic form and a solvate of glibenclamide. Acta Pharm Hung. 1997; 67:81-8.

7. Panagopoulou-KaplanI A, Malamataris S. Preparation and characterization of a new insoluble polymorphic form of glibenclamide. Int. J. Pharm. 2000 ; 19:239-46.

8. Rodríguez MS, Luna J, Carlucci A, Bregni C. Relación de estabilidad termodinámica relativa entre polimorfos de glibenclamida. Acta Farm Bonaer. 2004 ; 23(2):169-75.

9. Cuffini SL, Pitaluga AJR, Tombari DG. Polimorfismo em Fármacos. In: Storpirtis S, Gonçalves JE, Chiann C, Gai MN. Biofarmacotécnica. Rio de Janeiro: Guanabara Koogan, 2009. p.21-29.

10. Neugebauer G, Betzien G, Hrstka V, Kaufman B, Vonmöllendorff E, Abshagen U. Absolute bioavailability and bioequivalence of glibenclamide (Semi-Euglucon N). Int J Clin Pharmacol Ther Toxicol. 1985;23(9):453-60.
11. Blume H, Ali SL, Siewert M. Pharmaceutical quality of glibenclamide products: Multinational post market comparative study. Drug Dev Ind Pharm. 1993; 19(20):2713-41.

12. United States Pharmacopeia. 32. ed. Rockville: United States Pharmacopeial Convention, v.1, p.549-552, 2009.

13. Aulton ME. Delineamento de Formas Farmacêuticas: 2.ed. Rio Grande do Sul: Artmed, 2005. p. 25.

14. Brasil. Agência Nacional de Vigilância Sanitária. Resolução RDC $\mathrm{N}^{\circ}$ 31, de 11 de agosto de 2010. Dispõe sobre a realização dos Estudos de Equivalência Farmacêutica e de Perfil de Dissolução Comparativo. Diário Oficial [da] República Federativa do Brasil. Brasília, DF, 12. Ago. 2010. No 154, p. 36-8

15. O'hara T, Dunne A, Butler J, Devane J. A review of methods used to compare dissolution profile data. Pharm Sci Technol Today. $1998 ; 1: 214-23$.

16. Zhang G, Law D, Schmitt E, Qiu Y. Phase transformation considerations during process development and manufacture of solid oral dos age forms. Adv Drug Deliv Rev. 2004; 56:371-90.

17. Brasil. Agência Nacional de Vigilância Sanitária. Resolução RDC No 134, de 29 de maio de 2003. Dispõe sobre a adequação dos medicamentos já registrados. Diário Oficial [da] República Federativa do Brasil. Brasília, DF, Jun. 2003. p.47-8. 